\title{
Laser-induced substructures in above-threshold-ionization spectra from intense few-cycle laser pulses
}

\author{
M. Wickenhauser, ${ }^{1,2}$ X. M. Tong, ${ }^{1,3}$ and C. D. Lin ${ }^{1}$ \\ ${ }^{1}$ J. R. Macdonald Laboratory, Physics Department, Kansas State University, Manhattan, Kansas 66506-2604, USA \\ ${ }^{2}$ Institute for Theoretical Physics, Vienna University of Technology, A-1040 Vienna, Austria \\ ${ }^{3}$ Institute of Materials Science, Graduate School of Pure and Applied Science, University of Tsukuba, 1-1-1 Tennodai, Tsukuba, \\ Ibaraki 305-8573, Japan and Center for Computational Sciences, University of Tsukuba, 1-1-1 Tennodai, Tsukuba, \\ Ibaraki 305-8577, Japan
}

(Received 4 November 2005; published 9 January 2006)

\begin{abstract}
We have calculated above-threshold-ionization (ATI) spectra for argon in a strong, few-cycle $400 \mathrm{~nm}$ laser pulse with an $a b$ initio solution of the time-dependent Schrödinger equation and by using a model based on the strong field approximation. We find additional peaks in the resulting ATI spectra which are not present for longer pulses. This substructure is due to the rapidly changing ponderomotive potential in the short laser pulse. It is sensitive to the pulse duration and the pulse envelope and is a general feature of ionization spectra originating from a short, intense laser pulse.
\end{abstract}

DOI: 10.1103/PhysRevA.73.011401

PACS number(s): $32.80 . \mathrm{Rm}, 32.80 . \mathrm{Fb}, 42.50 . \mathrm{Hz}$

In recent years, the phenomenon of above-thresholdionization (ATI) has been extensively studied with femtosecond laser pulses $[1,2]$. In this process an atom absorbs more photons than the minimum number required for ionization $[3,4]$. The overall characteristics of ATI spectra are peaks separated by the photon energy and shifted by the ponderomotive potential. However, the concept of the ponderomotive potential, which is the cycle averaged kinetic energy of an electron in an oscillating field, is questionable in the fewcycle regime [5]. Since the field rises from zero to its peak value within a few femtoseconds, each cycle has a corresponding ponderomotive potential. The question arises as to how well ATI spectra originating from intense few-cycle pulses resemble the well-known ATI structure by the longer pulses. Recently, in Ref. [6] it has been shown that the ionization of atoms with few-cycle pulses makes a qualitative difference to ionization with longer pulses, since the pulse duration is shorter than the Kepler period of most Rydberg states. Resonancelike features are therefore suppressed for the shorter pulses.

The goal of this work is to understand and analyze a certain substructure which we found in the ATI spectra of argon. The results presented in this paper are from an $a b$ initio solution of the time-dependent Schrödinger equation (TDSE) and by using a model based on strong field approximation (SFA) [7]. The TDSE is solved for a model argon atom [8] in the single active electron approximation with a generalized pseudo spectral method in the energy representation. The detailed procedure can be found in Refs. [9,10]. Atomic units $\left(m_{e}=\hbar=e=1\right)$ are used throughout the paper unless otherwise indicated. Although we focus in this work on argon, the discussed structure is universal and is also theoretically observed in the ionization spectra of neon, hydrogen, and $\mathrm{H}^{-}$.

We use a 10 fs full width at half maximum (FWHM), linearly polarized laser pulse with a carrier wavelength of $400 \mathrm{~nm}$ and a peak intensity of $1.7 \times 10^{14} \mathrm{~W} / \mathrm{cm}^{2}$. The electric field of the laser pulse can be written in the form $\mathbf{F}(t)$ $=F_{0} \hat{\epsilon} a(t) \cos (\omega t+\varphi)$, with $\hat{\boldsymbol{\epsilon}}$ the polarization vector, $\omega$ the carrier frequency, and $\varphi$ the carrier-envelope phase. The envelope function $a(t)$ is chosen to be

$$
\sin ^{2}\left(\frac{\pi(t+\tau / 2)}{\tau}\right) \quad-\frac{\tau}{2}<t<\frac{\tau}{2}
$$

and zero elsewhere. In the model calculation the momentumdependent ionization amplitude is given by [7]

$$
b(\mathbf{p})=i \int_{-\infty}^{\infty} d t e^{i \phi_{V}(\mathbf{p}, t)}\left\langle\mathbf{p}-\mathbf{A}(t)\left|H_{L}(t)\right| g\right\rangle_{t},
$$

where $H_{L}(t)=\mathbf{F}(t) \mathbf{z}$ is the dipole operator. The polarization axis is along the $z$ direction. We have calculated the dipole moment using the correct energy scaled hydrogenic $3 p$ wave function. The Volkov phase in Eq. (1) can be written as

$$
\phi_{V}(\mathbf{p}, t)=-\int_{t}^{\infty} d t^{\prime} \frac{\left[\mathbf{p}-\mathbf{A}\left(t^{\prime}\right)\right]^{2}}{2} .
$$

In Fig. 1 we present the photoelectron spectra obtained by an $a b$ initio solution of the TDSE (lower curve) and for comparison by using the SFA (upper curve). We have observed a good agreement between the two calculations which allows us to use the simple SFA model for the further analysis of the substructure.

Three ATI peaks which are spaced by the photon energy $\omega=3.1 \mathrm{eV}$ can be seen in the spectrum. On the right side each ATI peak breaks up into a number of smaller subpeaks with varying amplitudes. Although these subpeaks in the ATI spectra look similar to Freeman resonances [11], here we prove that they are of a different nature. The inset in Fig. 1 shows the spectra in the neighborhood of the first ATI peak for three increasing intensities, 1.7, 1.75, and 1.8 $\times 10^{14} \mathrm{~W} / \mathrm{cm}^{2}$, respectively, obtained by solving TDSE. The overall structure is identical for all three intensities. However, the peak positions differ. Apart from ionizing the atom, the laser field also induces an ac Stark shift to the atomic level. This shift increases the ionization potential by the pon- 


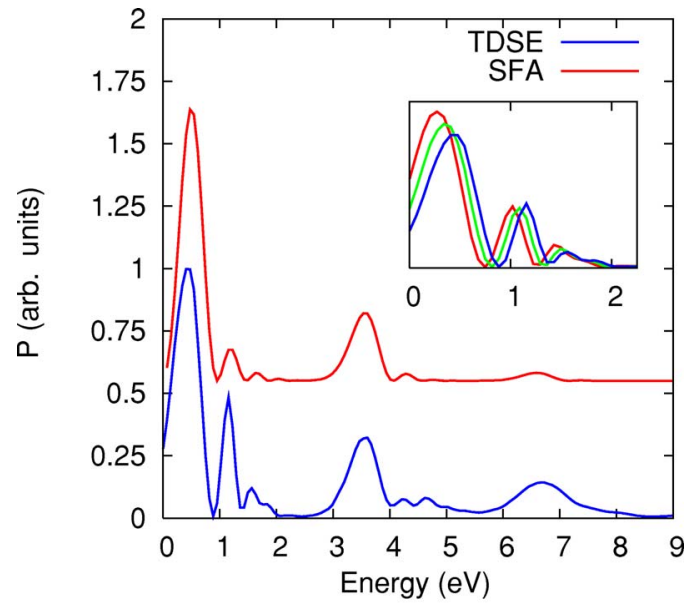

FIG. 1. (Color online) Photoelectron spectra of argon calculated by solving the TDSE and by using a model based on SFA. We normalize the SFA calculation to the same maximum amplitude as the TDSE result and add a constant to distinguish the two lines more clearly. Each of the three ATI peaks is separated into a number of smaller peaks with varying amplitudes. Laser parameters are $\omega$ $=400 \mathrm{~nm}, I=1.7 \times 10^{14} \mathrm{~W} / \mathrm{cm}^{2}$. The pulse length $(\mathrm{FWHM})$ is $10 \mathrm{fs}$ Inset: Photoelectron spectra in the region of the first ATI peak for three different intensities, 1.7 (blue), 1.75 (green), and 1.8 (red) $\times 10^{14} \mathrm{~W} / \mathrm{cm}^{2}$, respectively, obtained by solving TDSE. The peak positions shift closer to the threshold with increasing intensities.

deromotive energy, $U_{p}=I /\left(4 \omega^{2}\right)$. Because of energy conservation the energy of an ATI peak originating from ionization with absorbing $n$ photons is given by

$$
E=n \omega-\left(I_{p}+U_{p}\right),
$$

where $I_{p}$ is the ionization potential of the unperturbed atom and $U_{p}$ is calculated from the peak laser intensity. According to this equation we can identify $\left(I_{p}+U_{p}\right)$ as the field-dressed ionization potential. Thus the ATI peak originating from the highest intensity pulse is shifted most to the left, since it experiences the largest field-dressed ionization potential. Since the energy positions of all the subpeaks in the ATI spectra move with changing intensity, they do not show the typical behavior of a Freeman resonance, where the energy position stays fixed $[11,12]$ when the laser intensity is varied.

Another proof of the nonresonant character of the small peaks is provided by a comparison of the TDSE calculation with the results obtained from the SFA. All main features from the numerical calculation, including ATI peaks, as well as subpeaks, can be reproduced with the SFA model. In this model, besides the ground state, only the continuum states are included; therefore, the small peaks cannot originate from any intermediate resonances. Furthermore, the similarity in the results between the two different calculations indicates that the effect of the long-range Coulomb force, which is neglected in the SFA, has only negligible influence on the substructure in the ATI spectrum.

We note that in this paper the presented spectra do not depend on the carrier envelope phase, as can be expected for a $10 \mathrm{fs}$ laser pulse. In the following we show that the substructure is caused by the changing field-dressed ionization

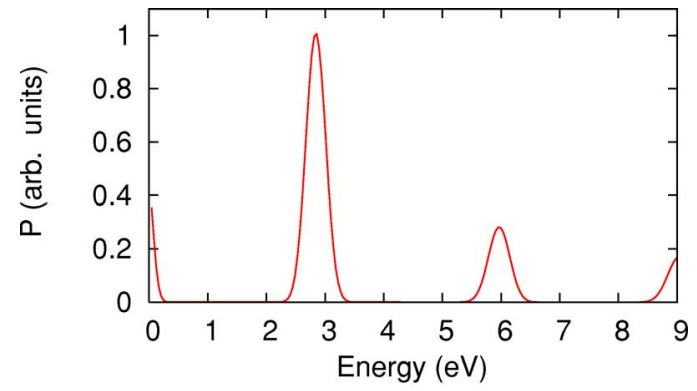

FIG. 2. (Color online) Photoelectron spectrum calculated using the same parameters as in Fig. 1 obtained within the SFA, but with the redefined Volkov phase $\widetilde{\phi}_{V}$ which does not include the ponderomotive energy shift. All subpeaks disappear from the spectrum. The peaks are shifted compared to Fig. 1 as a result of the missing ponderomotive energy.

potential during the ionization process. It is significant for short, intense laser pulses and is therefore a general feature which does not depend on the atomic target.

As seen from Eq. (4) the ponderomotive energy shifts the position of an ATI peak. Since in a few-cycle laser pulse the intensity changes rapidly in a relatively short time period, the contributions to ionization from different cycles of the pulse each observe a different ponderomotive potential and are thus not centered at the same energy. Therefore the ionization amplitudes from different cycles do not add up fully coherently while forming an ATI peak. The contribution coming from the region around the peak laser field is most shifted to the lower energy side compared to ionization from regions of the pulse with smaller intensity. These are shifted to the right side. Since a laser with peak intensity of 1.7 $\times 10^{14} \mathrm{~W} / \mathrm{cm}^{2}$, as we have used for our simulations and which is a typical experimental value, has a ponderomotive energy of $2.55 \mathrm{eV}$ at $400 \mathrm{~nm}$ compared to a spacing between the ATI peaks of $3.1 \mathrm{eV}$, this shift cannot be neglected.

For a better understanding we have a closer look at equation (1). The term $\mathbf{A}(t)^{2} / 2$ in the Volkov phase averaged over one cycle equals the ponderomotive potential and acts as a time-dependent energy shift. To prove our assumption that the small peaks are caused by this time dependent shift we subtract the corresponding term from the Volkov phase so that

$$
\widetilde{\phi}_{V}(\mathbf{p}, t)=-\int_{t}^{\infty} d t^{\prime}\left(\frac{\mathbf{p}^{2}}{2}-\mathbf{p} \mathbf{A}\left(t^{\prime}\right)\right)
$$

remains. Figure 2 shows the results obtained with the model when using $\widetilde{\phi}_{V}$ as the phase factor. All other parameters are the same as in Fig. 1. Obviously, all the substructure in the ATI spectrum disappears. The ATI peaks are shifted with respect to Fig. 1 since the ponderomotive shift is missing.

In Fig. 3(a) we present the spectrum originating from ionization from only one optical cycle at three different positions in the laser pulse. This calculation was performed within the SFA since there we can easily distinguish between ionization, which is described by the dipole matrix element, and the propagation of the ionized electrons which is repre- 


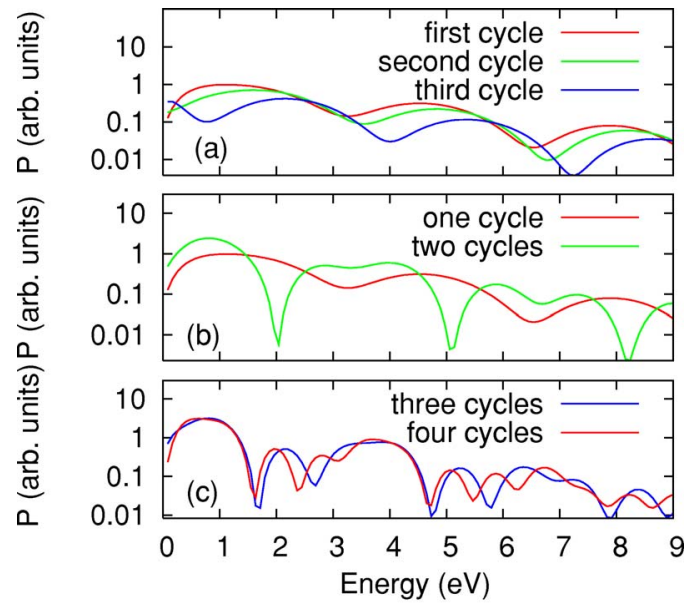

FIG. 3. (Color online) (a) Spectra originating from ionization from one single optical cycle at three different periods of the laser pulse, $[0, T],[T, 2 T]$, and $[2 T, 3 T]$, respectively. Note the logarithmic scale. The contributions from the different cycles are shifted relatively to each other according to the different ponderomotive potential at the time of ionization. (b) Spectra resulting from ionization through one, and two optical cycles, respectively, starting at the peak laser field. (c) Spectra resulting from ionization through three and four optical cycles, respectively. The number of peaks in the ATI spectra increases with the number of laser cycles relevant for the ionization process. Calculations carried out within the SFA.

sented by the Volkov phase factor. The three curves in Fig. 3 (a) originate from ionization only during the intervals $[0, T],[T, 2 T]$ and $[2 T, 3 T]$, respectively, where $T=1.33 \mathrm{fs}$ is the laser period. (At time $t=0$ in our simulation the laser pulse has reached its peak intensity.) After ionization we propagate each time until the pulse is over.

Obviously, ionization from one optical cycle [Fig. 3(a)] is already enough to observe the typical ATI structure in the spectrum, though the peaks are broader than they will become after ionization from the whole pulse. The peak positions from the contributions of the different cycles are shifted accordingly to the instantaneous field-dressed ionization potential at the time of release of an electron. Also visible is the decreasing probability for ionization from the different cycles. Figures 3(b) and 3(c) show the spectrum from ionization of $t=1,2,3$, and 4 optical cycles, respectively. While the absolute position and number of peaks obviously depend strongly on the phase with which the ionization amplitudes from the different cycles interfere, we note that a general trend can be seen: The number of peaks increases with the number of cycles relevant for ionization and is therefore an indicator for the pulse length.

Another interesting property of these peaks is presented in Fig. 4. In this figure we compare the energy positions of the different subpeaks as a function of intensity. The peak positions have been determined from numerical solutions of the TDSE for the corresponding intensities. While the shift of the main peak agrees well with the ponderomotive potential calculated at the peak laser field, the shift of the smaller peaks is getting less, as clearly seen from their decreasing slopes. The energy shift is a measure of the average intensity which is present during the ionization process which contrib-

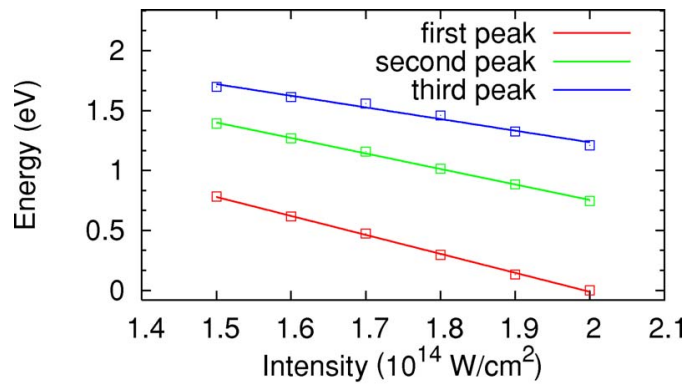

FIG. 4. (Color online) Energy positions of the three leftmost peaks in Fig. 1 as a function of peak laser intensity. The shift of the leftmost peak (lowest line) agrees well with the ponderomotive energy calculated with the peak laser intensity. The shifts of the following subpeaks are less due to the smaller intensity the electrons experience during the ionization process.

utes most to a certain peak. Thus the decreasing shift follows approximately the envelope function of the laser pulse.

We have performed simulations for longer pulse durations. In both the numerical as well as the model calculation, we see an increase in the number of subpeaks with the pulse duration. Within the SFA all subpeaks remain separately visible. However, in the numerical calculation we observe a different trend. As the spacing between the various peaks becomes narrower with increasing pulse length, the intermediate regions begin to fill up. Since the main difference between the SFA and the numerical calculation is the absence of the Coulomb potential in the former, we suspect that the Coulomb force, which leads to a phase correction [13] in Eq. (2), is responsible for this behavior. This observation is also in agreement with experimental data and calculations for longer pulses which do not show the discussed structure in the ATI spectra.

In Fig. 5 the ATI spectrum for a $10 \mathrm{fs}, 800 \mathrm{~nm}$ pulse is presented. Again we compare the TDSE with the SFA calculation, though this time we do not observe a good agreement. The difference between the two results probably can be attributed to Freeman resonances which are not included in the

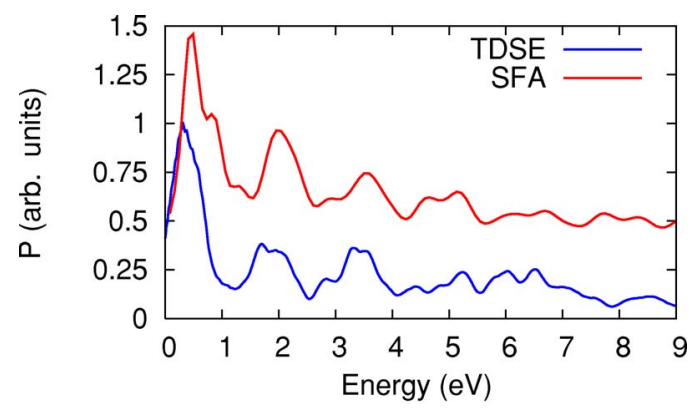

FIG. 5. (Color online) Photoelectron spectra of argon calculated by solving the TDSE and by using SFA for a $10 \mathrm{fs,} 800 \mathrm{~nm}$ pulse with $2 \times 10^{14} \mathrm{~W} / \mathrm{cm}^{2}$ peak intensity. Compared to the $400 \mathrm{~nm}$ case in Fig. 1, the characteristic ATI peaks are still visible, but the spectrum shows a more complex structure. Differences between TDSE and the model calculation originate from the presence of Freeman resonances in the former. We normalize the SFA result to the same maximum amplitude as the TDSE calculation and add a constant to distinguish the two lines more clearly. 
SFA model. There are two reasons why this effect is more pronounced for $800 \mathrm{~nm}$ compared to the $400 \mathrm{~nm}$ case: Because of the smaller photon energy, the number of photons participating in the ionization process is doubled and therefore the chance of hitting a resonance increases. Secondly, the ponderomotive potential at the same intensity is larger by a factor of four which makes it easier to shift an atomic state into resonance. Additionally, the ATI peaks are more closely spaced so that even within the model calculation, the substructure is not so clearly visible in the $800 \mathrm{~nm}$ spectrum since the small peaks from one ATI peak could appear at the position of the next ATI peak. We summarize that the discussed substructure is also expected in the $800 \mathrm{~nm}$ case. However, it is almost impossible to separate it from Freeman resonance contributions.

In conclusion, we have investigated the ATI spectra for argon by short laser pulses, both by solving the timedependent Schrödinger equation and with a model based on SFA. We have observed and analyzed a new class of subpeaks in the ATI spectra which is caused by the rapidly changing envelope of the laser field in a short pulse. The field-dressed ionization potential of the atom follows the rise and fall of the pulse envelope, and thus ionization at different optical cycles is mapped onto correspondingly different energies. A similar time to energy mapping in the laser field has been observed in experiments by the group of Krausz. In their case, an extreme ultraviolet attosecond pulse ionized the electron and a femtosecond laser pulse was used as a probe $[14,15]$. In both experiments the intensity of the laser was about two orders of magnitude lower than the one used here so that the change in the ponderomotive potential was negligible. The change in the electron spectra in their experiments originates mainly from the factor $\mathbf{p A}(t)$ in the Volkov phase. In contrast, we have reported in this paper on the influence of the time-dependent ponderomotive potential on the ionization process. The time-to-energy mapping occurs by shifting the field-dressed ionization potential of the atomic system itself on a femtosecond time scale.

This work was supported in part by Chemical Sciences, Geosciences and Biosciences Division, Office of Basic Energy Sciences, Office of Science, U.S. Department of Energy. M.W. would like to thank the Austrian Academy of Sciences for financial support.
[1] M. J. Nandor, M. A. Walker, L. D. VanWoerkom, and H. G. Muller, Phys. Rev. A 60, R1771 (1999).

[2] R. Wiehle, B. Witzel, H. Helm, and E. Cormier, Phys. Rev. A 67, 063405 (2003).

[3] P. Agostini, F. Fabre, G. Mainfray, G. Petite, and N. K. Rahman, Phys. Rev. Lett. 42, 1127 (1979).

[4] L. F. DiMauro and P. Agostini, Advances in Atomic, Molecular and Optical Physics (Academic Press, New York, 1995), Vol. 35.

[5] F. Lindner et al., Phys. Rev. Lett. 95, 040401 (2005).

[6] F. Grasbon et al., Phys. Rev. Lett. 91, 173003 (2003).

[7] M. Lewenstein, P. Balcou, M. Y. Ivanov, A. L'Huillier, and P. B. Corkum, Phys. Rev. A 49, 2117 (1994).
[8] X. M. Tong and C. D. Lin, J. Phys. B 38, 2593 (2005).

[9] X. M. Tong and S.-I. Chu, Chem. Phys. 217, 119 (1997).

[10] X. M. Tong and S.-I. Chu, Phys. Rev. A 61, 031401(R) (2000).

[11] R. R. Freeman, P. H. Bucksbaum, H. Milchberg, S. Darack, D. Schumacher, and M. E. Geusic, Phys. Rev. Lett. 59, 1092 (1987).

[12] G. D. Gillen and L. D. VanWoerkom, Phys. Rev. A 68, 033401 (2003).

[13] C. C. Chirilă and R. M. Potvliege, Phys. Rev. A 71, 021402(R) (2005).

[14] M. Drescher et al., Nature (London) 419, 803 (2002).

[15] E. Goulielmakis et al., Science 305, 1267 (2004). 